\title{
Solid Bare Strange Quark Stars
}

\author{
Renxin Xu \\ School of Physics, Peking University, Beijing 100871, China
}

\begin{abstract}
The reason we need the terms "strange", "bare" and "solid" before quark stars is presented concisely, although some fundamental issues are not certain. Observations favoring these stars are introduced.
\end{abstract}

\section{Hadronic Stability: Why Strange?}

Strange (quark) stars are quark stars with strangeness. What is strangeness? Some peculiar cosmic ray events were discovered in 1947, with a strange nature of "being produced rapidly in pairs, but decaying slowly independently". These particles were thus called "strange" particles, and a new quantum number, "strangeness" $(S)$, was introduced. In the standard model of particle physics, it is known that the strangeness represents actually the existence of a new kind of quarks, the strange quark, s. In this model, a neutron is composed of three valence quarks (one up and two down quarks), three quark pairs $(u \bar{u}, d \bar{u}, s \bar{s})$, and gluons, i.e., $n=\{u d d, u \bar{u} d \bar{d} s \bar{s}, g\}$; a proton $p=\{u u d, u \bar{u} d \bar{d} s \bar{s}, g\}$. A neutron (or proton) does not have strangeness because of $S(s)=-1$ and $S(\bar{s})=1$. Hadrons with three valence quarks are called baryons (e.g., protons and neutrons).

And then, how to produce strangeness in a star if it is the remains of an evolved main sequence star? Atomic nuclei in normal stars are made only of the two nucleons (proton and neutron), without strangeness. Two scenarios are outlined for creating strangeness in dense stellar matter. (1) The first one is in the hadronic degrees of freedom (i.e., hadrons as quasiparticles). Hyperons are baryons with strangeness, in which one or more valence quarks are replaced by $s$-quarks (e.g., $\Lambda=\{u s d\}, \Omega=\{s s s\}$ ). Nucleons with high enough Fermi energy (e.g., in traditional neutron stars) may decay into hyperons by weak interactions. Such nuclear matter with strangeness is called strange hadronic matter, and the corresponding neutron stars are named hyperon stars. The study of hypernuclei may help us to understand hyperon stars. (2) The second one is in the quark degrees. Suppose that quarks within hadrons are deconfined in high density (to form quark-gluon plasma, or called quark matter), one may expect that twoflavor quark matter (i.e., $u$ and $d$ ) appears in "neutron" stars. However, in case of $s$-quark mass being smaller than the Fermi energy of $u$ - and $d$-quarks, the system may become more stable via weak-interaction, decaying into three-flavor quark matter. A further radical speculation is that bulk three-flavor quark matter is absolutely stable (the Bodmer-Witten conjecture); strange stars are accordingly made of such strange quark matter (SQM) with nearly equal numbers of the light quarks. Note: a SQM core and outer nuclear matter may coexist over macroscopic scale if bulk SQM is metastable (mixed stars).

And then, can hadrons (e.g., neutrons) in "neutron" stars be deconfined? The underlying theory of the interaction between quarks is believed to be quan- 
tum chromodynamics (QCD), based on which it is proved that the interaction is asymptotically free in the smaller scale (another property of QCD could be color confinement in the larger scale, which has not been proved yet). A short distance on average between quarks is possible by two methods: (a) creating virtual quark pairs in relativistic heavy ion collisions, (b) crowding hadrons in compact stars. The former is of temperature-dominated, and the later baryondensity-dominated. Therefore, possible quark-deconfinement in compact stars might be a straightforward consequence of asymptotic freedom.

In summary, the existence of strange stars (SSs) could be quite natural if bulk SQM is an absolutely stable state of three-flavor color interaction system, though heavy quark (charm, top, and bottom quarks) degrees could be excited if the chemical potential is much high (e.g., in a SS core where the density is very high). It is still not certain whether observed pulsar-like stars are neutron or strange stars. No solid evidence for pulsars being neutron stars (NSs) yet exists (though many authors believe this conventionally)!

\section{Formation and Evolution: Why Bare?}

Because of the significant mass differences between $s$ - and $u$ - (or $d$-) quarks, electrons in SQM maintain charge neutrality. The electrons occupy a larger space $\left(\sim 10^{3} \mathrm{fm}\right)$ than that of quarks since the electromagnetic interaction is weaker than the color one. This results in a strong magnetic field $\left(\sim 10^{17} \mathrm{~V} \mathrm{~cm}^{-1}\right)$ just above the quark surface. The field could repulse (or support) positively charged nuclei, and a crust with mass $\sim 10^{-5} \mathrm{M}_{\odot}\left(\sim 10^{2} \mathrm{~m}\right.$ thickness $)$ may cover the SQM. There is no difference between the surfaces of crusted SSs and NSs.

With regard to the existence of SSs, radio pulsars were not thought of as bare SSs (BSSs) until Xu \& Qiao (1998) showed that BSSs can function as radio pulsars, with three advantages. Can the quark surface be exposed to the cosmos? If it can, the quark surface could be used to identify a SS. (1) A crust can hardly survive the detonation flame during a combustion of nuclear matter into quark matter, because of the rapid energy release and high temperature. (2) Unless the spin period is $>10^{3} \mathrm{~s}$ and the accretion rate is higher than Eddington, an isolated SS could not be covered by a crust through accretion during its lifetime.

\section{Their Astrophysical Appearances: Why Solid?}

Condensation in momentum space (e.g., 2SC, CFL, LOFF states) is currently focused on in the study of quark matter with high density but low temperature. However, can condensation in position space occur? No such competition (i.e., condensation in momentum vs. position spaces) happens in the electric superconductivity due to the strong Coulomb repulsion between electrons.

Quantum effects dominate in an ideal gas if the thermal de Broglie wavelength is larger than the mean distance between particles, but the case is different if strong interactions participate. The interaction may favor a condensation in position space (Xu 2003), which results in the formation of $n$-quark clusters in SQM (Note: these clusters are not bag-like color singlet hadrons; if so, the Bodmer-Witten conjecture is violated). The mean distance between $n$-quark clusters is $l \sim\left[n /\left(3 n_{\mathrm{b}}\right)\right]^{1 / 3}$ for SQM with density $n_{\mathrm{b}}$. The distance is $l \sim 1 \mathrm{fm}$

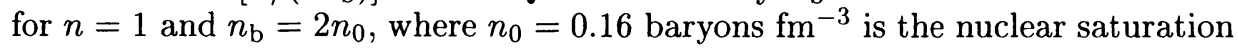


density. The interaction between quark clusters could be of a well potential, the depth of which should be $V_{0}>\sqrt{(\hbar c / l)^{2}+m^{2} c^{4}}-m c^{2}$, with $m$ the mass of the clusters, in order to describe classically the cluster gas. Furthermore, if the thermal excitation is not enough, $\sqrt{(\hbar c / l)^{2}+m^{2} c^{4}}-m c^{2}+k T \ll V_{0}$, with $T$ the temperature, the clusters might be localized to form a solid state. In the nonrelativistic (NR) approximation, this means $\hbar^{2} /\left(2 m l^{2}\right)+k T \ll V_{0}$. Assuming the interaction is described by the Lennard-Jones potential, $V(r)=-A / r^{6}+B / r^{12}$, we have $l=(2 B / A)^{1 / 6}$ and $V_{0}=-V(l)=A^{2} /(4 B)$. Therefore the condition for a solid state of SQM in the NR case is $A^{2} /(4 B)-\hbar^{2} /(2 m)(A / 2 B)^{1 / 3}-k T \gg 1$.

Recent experimental evidence for multi-quark $(n>3)$ particles (e.g., Bai et al. 2003), although still not being understood in QCD (e.g., Jaffe \& Wilczek 2003), may increase the possibility of quark clustering in SQM. These multiquark hadrons may decay rapidly by strong interaction; nonetheless, such clusters could be stable in SSs since their decay into hadrons would be suppressed or forbidden if the Bodmer-Witten conjecture is correct.

Actually, there may be a few observational hints of such a solid quark state.

Thermal spectra without atomic features: Atomic lines are not detected definitely yet in thermal radiation ( $\mathrm{Xu} 2002$ ). It is natural to understand the thermal spectra observed in a model of SSs with solid quark surfaces, where the thermal radiation could be analogous to that of metals, for RX J1856.5-3754 (Zhang et al., these proceedings) as well as for other sources.

There are, in fact, some efforts to understand the thermal spectra in the conventional model of NS atmospheres. (1) Featureless spectra were suggested as an indication of the vacuum polarization effect (Ho \& Lai 2003), but a satisfactory fit is not obtained with the inclusion of the polarization effect. (2) A hypothetical plasma phase condensation transition was suggested (Lai \& Salpeter 1997) for NS atmospheres with high $B$ or low $T$, and was applied, though not very successfully, to interpret the X-ray spectrum of RX J1856.5-3754 (Turolla, Zane \& Drake 2004). However a consistent study of the phase transition in thermodynamics is still not done yet. (3) Rapid rotating of a NS may smear a spectral line, but such a NS may hardly become radio "dead" (i.e., below the death line). Additionally, a problem could be inherent in all of these efforts: How to calm down the magnetospheric activities (e.g., AXP/SGR-like persistent and burst $\mathrm{X}$-ray emission, radio emission due to pair-plasma instabilities, etc.) for such a NS with strong field or high spin frequency?

Pulsar glitching and free-precession: The current model for glitches involves neutron superfluid vortex pinning and the consequent fluid dynamics. However, the pinning should be much weaker than predicted by the model, at laest for two radio pulsars (PSRs B1828-11 and B1642-03), otherwise the vortex pinning will damp out the precession on timescales much smaller than observed. In addition, the picture that a NS core contains coexisting neutron vortices and proton flux tubes is also inconsistent with observations of freely precessing pulsars (Link 2003). Theoretically, a definitive conclusion on the nature of vortex pinning has not been reached yet due to various uncertainties in the microscopic physics. This discrepancy could be circumvented if radio pulsars are solid: there is no damping in free-precession of solid stars, and glitches reflect the behaviors of global starquakes. The stresses, which trigger a starquake, develop due to spindown or, possibly due to the frame-dragging effect. 
The global starquake can also result in an exponential recovery of postglitches. For a solid star, the angular frequency, $\Omega(t)$ and the moment of inertia, $I(t)$, as functions of time, $t$, are governed by $\{\mathrm{d}(I \Omega) / \mathrm{d} t=-\alpha, \dot{I}=$ $\left.-\kappa\left(I-I_{0}\right), I_{0}=I_{0}(\Omega)\right\}$, where $\alpha$ is the external braking torque which is known for a star with certain magnetic momentum, $I_{0}$ is the inertia moment of the star in force-free equilibrium (i.e., no stress), and the recovery of the inertial moment, $I$, is assumed to be at a rate being proportional to $\left(I-I_{0}\right)$. These equations are closed if the function $I_{0}(\Omega)$ is given, which can be well approximated by calculating the Maclaurin configurations since the density of a SS with mass $<1.4 \mathrm{M}_{\odot}$ is almost uniform. If we temporarily neglect $\alpha, I_{0}$ is a constant. One then has $\delta I \equiv I-I_{0}=-\Delta I \exp [-\kappa t]$ from the second equation, if a glitch occurs at $t=0$, with $\Delta I$ the initial departure of inertia moment after the glitch (Note: $\left.I(0)<I_{0}\right)$. Therefore we have glitch recovery behavior of the form $\Omega(t)-\Omega(0) \sim \exp [-\kappa t]$, the postglitch relaxation observed. It is worth noting that the superfluid vortex pinning and unpinning could also work during a starquake if, besides quark-clusters localized, superfluid free quarks exist too.

Others: (1) Pulsars with submillisecond spin periods? Rotating fluid stars are subject to the $r$-mode instability, which results in temperature-dependent minimum spin periods for SSs (Madsen 2000), but a solid star can spin more quickly. Solid SSs can be identified if pulsar spin frequencies are discovered beyond the $r$-mode critical value. (2) Starquake-induced magnetic reconnection or the strange planet's collision could be responsible for the bursting X-ray radiation of AXP/SGRs, while accretion results in their persistent X-ray emission.

\section{Conclusions}

The time for "neutron" star study has not passed, although it has been over 70 years since the related idea appeared. Such a compact star may not just be boring big "nuclei", but could be composed by matter of a new state: quarkgluon plasma. Recent observations challenge the conventional NS models, and should reveal valuable information on the quark matter state.

Acknowledgments. This work is supported by NSFC (10273001) and the Special Funds for Major State Basic Research Projects of China (G2000077602).

\section{References}

Bai, J. Z. et al. 2003, Phys. Rev. Lett., 91, 022001

Jaffe, R., \& Wilczek, F. 2003, Phys. Rev. Lett., 91, 232003

Ho, W. C. G., \& Lai, D. 2003, MNRAS, 338, 233

Lai, D., \& Salpeter, E. E. 1997, ApJ, 491, 270

Link, B. 2003, Phys. Rev. Lett., 91, 101101

Madsen, J. 2000, Phys. Rev. Lett., 85, 10

Turolla, R., Zane, S., \& Drake, J. 2004, ApJ, 603, 265

Xu, R. X., \& Qiao, G. J. 1998, Chin. Phys. Lett., 15, 934

Xu, R. X. 2002, ApJ, 570, L65

- 2003, ApJ, 596, L59 